\title{
Antioxidant Compound Extraction from Maqui (Aristotelia chilensis [Mol] Stuntz) Berries: Optimization by Response Surface Methodology
}

\author{
Issis Quispe-Fuentes ${ }^{1, *}$, Antonio Vega-Gálvez ${ }^{1}$ and Víctor H. Campos-Requena ${ }^{2}$ \\ 1 Department of Food Engineering, University La Serena, Raúl Bitrán 1305, Box 599, La Serena, Chile; \\ avegag@userena.cl \\ 2 Department of Polymers, Faculty of Chemical Sciences, University of Concepcion, Edmundo Larenas 129, \\ Casilla 160-C, Concepción, Chile; vcamposr@udec.cl \\ * Correspondence: iquispe@userena.cl; Tel.: +56-51-233-4808
}

Academic Editors: Farid Chemat and Maryline Abert-Vian

Received: 22 November 2016; Accepted: 29 January 2017; Published: 2 February 2017

\begin{abstract}
The optimum conditions for the antioxidant extraction from maqui berry were determined using a response surface methodology. A three level D-optimal design was used to investigate the effects of three independent variables namely, solvent type (methanol, acetone and ethanol), solvent concentration and extraction time over total antioxidant capacity by using the oxygen radical absorbance capacity (ORAC) method. The D-optimal design considered 42 experiments including 10 central point replicates. A second-order polynomial model showed that more than $89 \%$ of the variation is explained with a satisfactory prediction (78\%). ORAC values are higher when acetone was used as a solvent at lower concentrations, and the extraction time range studied showed no significant influence on ORAC values. The optimal conditions for antioxidant extraction obtained were $29 \%$ of acetone for 159 min under agitation. From the results obtained it can be concluded that the given predictive model describes an antioxidant extraction process from maqui berry.
\end{abstract}

Keywords: antioxidant activity; D-optimal design; Maqui; ORAC value

\section{Introduction}

Maqui (Aristotelia chilensis [Mol]Stuntz) is a native South America evergreen shrub. Particular attention has been paid to polyphenols and anthocyanins content in this berry, not only for its use as natural colorant, but also for its potential beneficial effects on human health, including the use as dietary supplements in functional food products [1,2]. The antioxidant capacity of maqui berry has been outstanding due to its high ORAC value, surpassing many fruits, i.e., blueberry, strawberry, raspberry, blackberry [3], Cape gooseberry and papaya [4], pomegranate, acai and cranberry [2]. This property relates maqui consumption to health benefits such as anti-diabetic and anti-inflammatory effects and the prevention of Alzheimer's disease [2]. Besides, berries have emerged as a rich dietary source of phenolic antioxidants and bioactive properties [5]. ORAC is currently the most relevant and most widely accepted method to determine antioxidant activity. Since the ORAC assay uses fluorescence for detection, it is ideal for the antioxidant analysis of red to blue colored anthocyanin rich extracts such as maqui berry extract [6].

Conventionally, the antioxidant compounds are extracted using a suitable solvent-followed-byevaporation method to separate the solvent from the product. The solubility and extractability of phenolic compounds in food matrices depend on solvent polarity, the nature of the polyphenols (e.g., degree of polymerization) and chemical properties of the phenolics, the composition of the food matrix and interactions between the compound and food matrix [7] besides being influenced by several 
extracting factors including temperature, solvent-to-sample ratio, particle size, time, the number of repeated extractions of the sample, as well as solvent type [8,9]. For a further valid comparison of the phenolic levels in berries and their derived products, it is necessary to use the same method of extraction and a comparative methodology for polyphenols quantification as well as its antioxidant capacity. The high variability of analytical methods used to quantify phenolic content is associated with sample extraction [10]. Finding new and safe antioxidants from natural sources is of great interest for applications in natural antioxidants, functional foods, and nutraceuticals [11], in addition to the application of a quick and low-cost technology. In the case of maqui berries, there is still limited available information concerning to an optimization of their antioxidant compounds.

The response surface methodology (RSM) establishes the relationship between a measured response and a variety of factors that influence an outcome [12]. Nwabueze [13] mentioned that importance to adapt the RSM as a mathematical model for bioprocess optimization is based on determining the optimum process variable combinations that maximize or minimize that product response. Thus, it is a useful tool for process optimization that allows measuring of independent variable influence on a response variable to be represented by a mathematical model that can reproduce the behavior of these parameters with a limited number of experiments [8]. Then, RSM is less laborious and time consuming than other approaches, and today is one of the most popular optimization techniques in the field of food science. Several authors have used RSM to assess the effect on the antioxidant capacity of leaves, fruits and food [14-16]. In order to provide a consistent data in the experimental region it is necessary to perform an optimal number of experiments under a correct design of experiments (DoE). When there are a high number of variables that must be studied or multilevel qualitative variables are present, D-optimal design is a useful choice to be used. D-optimal design is a computer-generated DoE that sets a minimal number of experiments from a pool of theoretically possible designs. It allows handling qualitative variables with two or more levels and quantitative variables at the same time. [17]. The application of D-optimal design has been reported in different areas including food technology [18-20], engineering [21], kinetic chemical reactions [22] and pharmaceutical field [23].

The objective of the current study was to assess the effect of three operating conditions of extraction process, namely solvent type (methanol, ethanol and acetone), solvent concentration and extraction time, on antioxidant capacity by ORAC method of the maqui berry extracts by using D-optimal design and RSM thus, a set of optimal extraction parameters can be achieved to obtain the highest values of ORAC.

\section{Methods}

\subsection{Materials}

The following reagents were used: 2,20-azobis-2-amidinopropane (AAPH) and fluorescein sodium salt were purchased by Merck (Merck, Germany). Organic solvent for analysis from Merck. Ultra-pure water was prepared by using a PW-Ultra Water System (Heal Force, Shanghai, China).

\subsection{Extraction Protocol}

Maqui berries (Aristotelia chilensis [Mol]Stuntz) were obtained from neighbouring forests in the town of Mulchen, Region Bio-Bio, Chile ( $37^{\circ} 43^{\prime} 08^{\prime \prime}$ S; $72^{\circ} 14^{\prime} 27^{\prime \prime}$ W). They were washed and their leaves were separated from other edible parts. The fresh berry was fragmented using an analytical mill (IKA®A-11, Wilmington, DE, USA) and immediately frozen with liquid nitrogen $(1: 2, w / v)$ to avoid the oxidation of the bioactive compounds. The fraction was homogenized and frozen to be used for the extracts.

One gram of finely ground maqui samples were added to $70 \mathrm{~mL}$ of solvents solution at different concentrations according to previous results described by Cacace and Mazza [24] introducing some modifications. The mixtures were then vigorously shaken $(145 \mathrm{rpm})$ and kept at $30^{\circ} \mathrm{C}$ in a water bath incubator (Memmert, WNB 22, Schwabach, Germany) for antioxidants extraction. After the evaluated 
extraction time, the resultant solutions were filtered through Whatman filter paper No.1. The extracts were evaporated (Multivapor Büchi P-6, Flawil, Switzerland) at $38{ }^{\circ} \mathrm{C}$ and redissolved in $10 \mathrm{~mL}$ methanol-formic acid (99:1). The extraction was stored at $-80^{\circ} \mathrm{C}$ until analyses (no longer than $48 \mathrm{~h}$ ).

\subsection{Determination of Antioxidant Capacity by ORAC Method}

The ORAC (Oxygen radical absorbance capacity) assay was performed following the procedure described by Zhang et al. [25]. $200 \mu \mathrm{L}$ of a fluorescein solution freshly prepared (100 nM, phosphate buffer, pH 7.4) was added to each well black plate and $40 \mu \mathrm{L}$ of extract solution or Trolox standard solution and then incubated at $37^{\circ} \mathrm{C}$ for $20 \mathrm{~min}$. The assay was initiated by adding the peroxyl radical generator AAPH (2,2'-Azobis[2-amidinopropane] dihydrochoride), prepared in phosphate buffer. Specifically, $35 \mu \mathrm{L}$ of AAPH, $0.36 \mathrm{M}$ were added and the fluorescence was read $\left(\lambda_{\text {ex }}=485 \mathrm{~nm}\right.$ and $\lambda_{\mathrm{em}}=535 \mathrm{~nm}$ ) every minute by using a Multilabel Plate Reader (Perkin-Elmer, Victor $\chi^{3}$, Hamburg, Germany) maintained at $37^{\circ} \mathrm{C}$ and the fluorescein loss was followed until the reading declined to over $95 \%$ of the initial reading. Standards and solution extracts were run in sextuple. Results for ORAC were determined by using the differences of areas under the fluorescein kinetic decay curve. The ORAC value of each solution extract was expressed in $\mu$ mol Trolox Equivalents/100 g sample ( $\mu \mathrm{mol} \mathrm{TE} / 100 \mathrm{~g})$.

\subsection{Design of Experiment and Statistical Analysis}

Based on the results of preliminary experiments, a three-level D-optimal design was conducted in this optimization study. Type of solvent $\left(x_{1}\right)$, concentration solvent $\left(\% v / v, x_{2}\right)$ and extraction time $\left(\min , x_{3}\right)$ were the independent variables selected to be optimized for the extraction of antioxidant compounds of maqui berry (see Table 1). ORAC value $(y)$ was selected as a dependent variable. A quadratic model was selected yielding an optimization design, whose statistical evaluation is described in Table 2. A second-order interaction model can represent the system by using the quadratic polynomial shown in Equation (1) that describes the impact of the three variables on the response.

$$
y=\sum_{i=1}^{k} b_{0}+\sum_{i=1}^{k} b_{i} x_{i}+\sum_{i=1}^{k} b_{i i} x_{i}^{2}+\sum_{j=i+1}^{k} b_{i j} x_{i} x_{j}+\varepsilon
$$

where $y$ is the measured response, $b_{0}$ is the constant term, $b_{\mathrm{i}}$ are the unknown regression coefficients of the variables $x_{\mathrm{i}}$ that must be determined, $b_{\mathrm{ii}}$ are the quadratic regression coefficients, $b_{\mathrm{ij}}$ are the interaction coefficients, and $\varepsilon$ are the residuals. The G-efficiency parameter (Table 2) compares the performance of the D-optimal design to that of a fractional factorial design and being its $100 \%$ implies that the model is equal to a fractional factorial design. It is recommended a G-efficiency over $60 \%-70 \%$ [26].

The selected D-optimal design consisted of 42 experiments given in Table 3 that includes a central point of 10 replicates used for variance calculation. ANOVA was performed for statistical validation of the regression at the $95 \%$ confidence level. Optimal variable conditions that maximize the response were calculated by RSM using the SIMPLEX method. MODDE $11^{\circledR}$ software (MKS Instruments AB, Umea, Sweden) was employed for ANOVA test, D-optimal design construction and RSM calculations.

Table 1. Variables and experimental levels for the D-optimal design.

\begin{tabular}{lccc}
\hline Qualitative Variables & \multicolumn{3}{c}{ Type } \\
\hline$x_{1}$ : Type of solvent & Methanol & Ethanol & Acetone \\
& & Level & High $(+1)$ \\
Quantitative variables & Low $(-1)$ & Medium $(0)$ & 100 \\
$x_{2}:$ Solvent concentration $(\% v / v)$ & 20 & 60 & 240 \\
$x_{3}:$ Extraction time $(\min )$ & 15 & 127.5 & Antioxidant capacity $(\mathrm{ORAC}) \mu \mathrm{mol} \mathrm{TE} / 100 \mathrm{~g}$ \\
\hline : Response variable & \multicolumn{4}{c}{} \\
\hline
\end{tabular}


Table 2. Statistical evaluation of the selected D-optimal design.

\begin{tabular}{lc}
\hline Candidate Set & \\
\hline Extreme vertices & 60 \\
Edge points & 60 \\
Centroids of high dimensional surface & 15 \\
Total runs & 135 \\
D-optimal & \\
Objective & Optimization \\
Model type & Quadratic \\
G-efficiency (\%) & 76.19 \\
Condition number & 4.88 \\
Design runs & 42 \\
\hline
\end{tabular}

Table 3. D-optimal design with experimental data.

\begin{tabular}{|c|c|c|c|c|}
\hline \multirow[b]{2}{*}{ Runs } & \multicolumn{3}{|c|}{ Variables } & \multirow{2}{*}{$\begin{array}{c}\text { Response } \\
y \\
\text { Antioxidant Capacity } \\
(\mu \mathrm{mol} T E / 100 \mathrm{~g})\end{array}$} \\
\hline & $\begin{array}{c}x_{1} \\
\text { Type of Solvent }\end{array}$ & $\begin{array}{c}x_{2} \\
\text { Solvent Concentration } \\
(\% v / v)\end{array}$ & $\begin{array}{c}x_{3} \\
\text { Extraction Time } \\
\text { (min) }\end{array}$ & \\
\hline 1 & methanol & $20(-1)^{\mathrm{a}}$ & $127.5(0)$ & $13,893.9$ \\
\hline 2 & methanol & $100(+1)$ & $127.5(0)$ & $11,870.4$ \\
\hline 3 & methanol & $60(0)$ & $15(-1)$ & $14,112.3$ \\
\hline 4 & methanol & $60(0)$ & $240(+1)$ & $13,445.0$ \\
\hline 5 & methanol & $20(-1)$ & $127.5(0)$ & $12,267.6$ \\
\hline 6 & methanol & $100(+1)$ & $127.5(0)$ & $11,720.2$ \\
\hline 7 & methanol & $60(0)$ & $15(-1)$ & $13,595.0$ \\
\hline 8 & methanol & $60(0)$ & $240(+1)$ & - b \\
\hline 9 & ethanol & $20(-1)$ & $127.5(0)$ & $11,574.7$ \\
\hline 10 & ethanol & $100(+1)$ & $127.5(0)$ & 5413.5 \\
\hline 11 & ethanol & $60(0)$ & $15(-1)$ & $15,931.4$ \\
\hline 12 & ethanol & $60(0)$ & $240(+1)$ & $13,179.7$ \\
\hline 13 & ethanol & $20(-1)$ & $127.5(0)$ & $10,572.9$ \\
\hline 14 & ethanol & $100(+1)$ & $127.5(0)$ & 5560.0 \\
\hline 15 & ethanol & $60(0)$ & $15(-1)$ & $12,438.1$ \\
\hline 16 & ethanol & $60(0)$ & $240(+1)$ & $13,179.7$ \\
\hline 17 & acetone & $20(-1)$ & $15(-1)$ & $18,920.9$ \\
\hline 18 & acetone & $100(+1)$ & $15(-1)$ & 4894.0 \\
\hline 19 & acetone & $20(-1)$ & $240(+1)$ & $17,275.7$ \\
\hline 20 & acetone & $100(+1)$ & $240(+1)$ & 5869.2 \\
\hline 21 & acetone & $20(-1)$ & $15(-1)$ & $16,240.9$ \\
\hline 22 & acetone & $100(+1)$ & $15(-1)$ & 4894.0 \\
\hline 23 & acetone & $20(-1)$ & $240(+1)$ & $17,275.7$ \\
\hline 24 & acetone & $100(+1)$ & $240(+1)$ & 5429.6 \\
\hline 25 & acetone & $20(-1)$ & $15(-1)$ & - \\
\hline 26 & acetone & $100(+1)$ & $15(-1)$ & 3419.5 \\
\hline 27 & acetone & $20(-1)$ & $240(+1)$ & $18,099.0$ \\
\hline 28 & acetone & $100(+1)$ & $240(+1)$ & 6796.7 \\
\hline 29 & acetone & $20(-1)$ & $15(-1)$ & $16,864.7$ \\
\hline 30 & acetone & $100(+1)$ & $15(-1)$ & 6796.7 \\
\hline 31 & acetone & $20(-1)$ & $240(+1)$ & $19,105.7$ \\
\hline 32 & acetone & $100(+1)$ & $240(+1)$ & 4817.3 \\
\hline 33 & methanol & $60(0)$ & $127.5(0)$ & $15,083.9$ \\
\hline 34 & methanol & $60(0)$ & $127.5(0)$ & $15,808.7$ \\
\hline 35 & methanol & $60(0)$ & $127.5(0)$ & $14,346.8$ \\
\hline 36 & ethanol & $60(0)$ & $127.5(0)$ & $15,060.1$ \\
\hline 37 & ethanol & $60(0)$ & $127.5(0)$ & $12,399.5$ \\
\hline 38 & ethanol & $60(0)$ & $127.5(0)$ & $16,240.9$ \\
\hline 39 & acetone & $60(0)$ & $127.5(0)$ & $17,668.9$ \\
\hline 40 & acetone & $60(0)$ & $127.5(0)$ & $14,692.5$ \\
\hline 41 & acetone & $60(0)$ & $127.5(0)$ & $14,571.1$ \\
\hline 42 & acetone & $60(0)$ & $127.5(0)$ & $18,796.5$ \\
\hline
\end{tabular}

${ }^{\mathrm{a}}$ codified levels of quantitative variables in parenthesis. ${ }^{\mathrm{b}}$ outliers or missing values. 


\section{Results and Discussion}

\subsection{Model Evaluation}

The effect of the three independent variables (type of solvent $\left(x_{1}\right)$, concentration solvent $(\%(v / v)$, $\left.x_{2}\right)$ and extraction time $\left(\min , x_{3}\right)$ ) on ORAC value $(y)$ was investigated. Response values were power transformed $\left(y^{2}\right)$ to achieve a fairly normal distribution of the data, thus improving its efficiency in the ANOVA analysis and also enhances the validity of the model. A quadratic polynomial model explains significantly $(p<0.05)$ maqui antioxidant capacity (ORAC value) regarding to the variables being studied. It was found that the ORAC value varied in wide ranges 3419.5-19,105.7 $\mu \mathrm{mol} \mathrm{TE} / 100 \mathrm{~g}$ depending on the changes of process variables. Gironés-Vilaplana et al. [4] reported highest value results within this range in Chilean maqui.

The parameters that evaluate the selected model are described in Table 4 . The goodness of fitted model was checked by the coefficient of determination $\left(R^{2}\right)$ which resulted 0.8992 indicating that the obtained model can explain $89.92 \%$ of variation in the antioxidant capacity value. It also shows a good prediction, where a goodness of prediction $Q^{2}=0.7801$ was obtained. Therefore, the model is sufficiently satisfactory to explain and predict the variation in the data based on capacity antioxidant of maqui berry (see Figure 1). The model validity value 0.5800 provides information on the lack of fit and indicates that a correct model type has been chosen. The value 0.8698 for reproducibility indicates a low pure error and a satisfactory experimental procedure represented in a small variation in the replicates [17]. The ANOVA for antioxidant capacity response $y$ indicates that the significance of the regression model is satisfactory $(p \leq 0.05)$ for this response. Table 5 shows that $F$-value was 22.706 and $p$-value of 0.000 imply that the model is significant. The insignificance $(p>0.05)$ on lack of fit for the model implies that this regression model is acceptable.

Table 4. Evaluation of the model.

\begin{tabular}{lc}
\hline Criteria & Value \\
\hline$R^{2}$ & 0.8992 \\
$Q^{2}$ & 0.7801 \\
Model validity & 0.5800 \\
Reproducibility & 0.8698 \\
Condition number $(n=40)$ & 4.10 \\
\hline
\end{tabular}

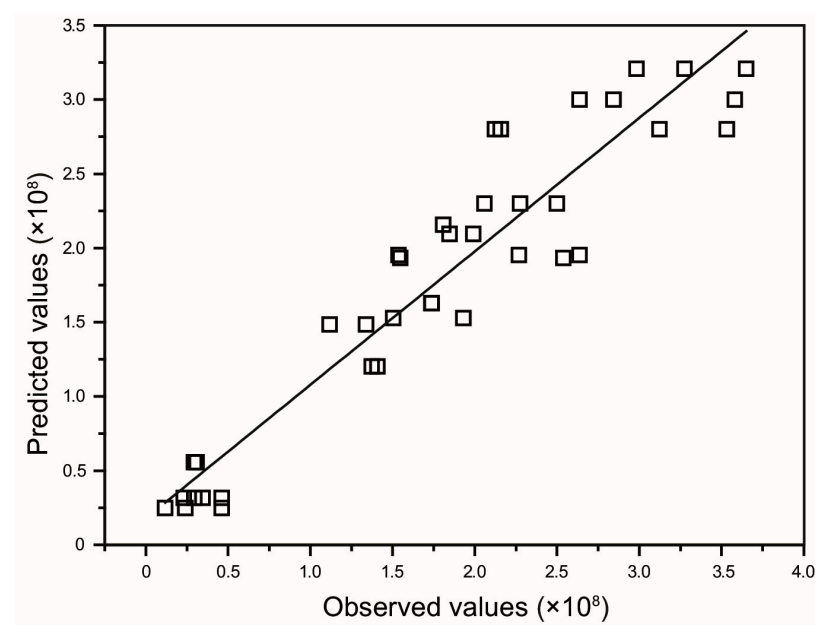

Figure 1. Plot of observed values vs. predicted values. Response values are power transformed $y^{2}$. $R^{2}=0.8992$. 
Table 5. ANOVA test for the chosen model.

\begin{tabular}{|c|c|c|c|c|c|c|}
\hline Source & Degrees of Freedom & Sums of Squares & Mean Squares & $F$-Value & $p$-Value & Standard Deviation \\
\hline Total corrected & 39 & $4.449 \times 10^{17}$ & $1.141 \times 10^{16}$ & & & $1.068 \times 10^{8}$ \\
\hline Regression & 11 & $4.001 \times 10^{17}$ & $3.637 \times 10^{16}$ & 22.706 & 0.000 & $1.907 \times 10^{8}$ \\
\hline Residual & 28 & $4.485 \times 10^{16}$ & $1.602 \times 10^{15}$ & & & $4.002 \times 10^{7}$ \\
\hline Lack of fit (model error) & 3 & $7.704 \times 10^{15}$ & $2.568 \times 10^{15}$ & 1.728 & 0.187 & $5.068 \times 10^{7}$ \\
\hline Pure error (replicate error) & 25 & $3.715 \times 10^{16}$ & $1.486 \times 10^{15}$ & & & $3.856 \times 10^{7}$ \\
\hline
\end{tabular}

\subsection{Model Interpretation}

Regression coefficients of the obtained model are presented as a graphical representation in Figure 2. The $p$-values were used as a tool to check the significance of each coefficient. Six significant terms have a large and significant $(p<0.05)$ effect on antioxidant capacity: two linear terms of solvent type ( $b_{1}$, ethanol, acetone) and solvent concentration $\left(b_{2}\right)$, the quadratic term of concentration $\times$ concentration $\left(b_{22}\right)$, and the interaction terms of methanol $\times$ concentration and acetone $\times$ concentration $\left(b_{12}\right)$. The other model terms, however, showed a non-significant effect $(p>0.05)$. The polynomial quadratic model for antioxidant capacity is given in Equation (2):

$$
y=b_{0}+b_{1} x_{1}+b_{2} x_{2}+b_{3} x_{3}+b_{12} x_{1} x_{2}+b_{13} x_{1} x_{3}+b_{23} x_{2} x_{3}+b_{22} x_{2} x_{2}+b_{33} x_{3} x_{3}
$$

The large and negative quadratic regression coefficient $b_{22}$ for concentration (green bar in Figure 2) indicates a strong curvature in the response for the antioxidant capacity (Figure 3), in which a maximum ORAC value can be reached within the experimental region. Regarding to solvent type, it is observed that only acetone has a positive effect in ORAC value (light blue bar in Figure 2). Thus, antioxidant compounds appear to be more extractable in acetone than in methanol or ethanol. Céspedes et al. [5] indicated that in maqui extract the acetone partition showed an excellent antioxidant activity.

It is known that the concentration of a solvent is an element affecting its polarity. In a sequence from the highest polarity to the lowest are: methanol, acetone and ethanol. In this way, a higher polarity compounds are responsible for maqui berry antioxidant capacity. The methanol proved to be a better solvent for the antioxidant capacity (DPPH) in strawberry [16] likewise this solvent is used for maqui ORAC value extraction by Gironés-Vilaplana et al. [4] for anthocyanin extraction and phenols from maqui by Brauch et al. [2], Rojo et al. [27] and Escribano-Bailón et al. [1]. Due to the higher polarity of methanol, more polar phenolic compounds may be extracted from maqui berry, such as several glycosides of anthocyanidins (delphinidin, cyanidin), flavonoids (quercetin, rutin, myricetin, catechin and epi-catechin) and phenolic acids (gentisic acid, ferulic acid, gallic acid, p-coumaric acid, sinapic acid, 4-hydroxybenzoic acid) [5].

As the acetone concentration in aqueous solution decreases, the solvent polarity increases. Therefore, it could be seen that a higher scavenging was attained by using more methanol concentration and acetone in aqueous solution. These results were in accordance with berries studies by Arancibia et al. [28] where results indicated that the bioactive compounds (polyphenols, flavonoids, flavanols, tannins and ascorbic acid) are significantly higher in water and significantly lower in non-polar solvent.

Maqui berry has a particularly high concentration of anthocyanins, and a prominent feature of the anthocyanin composition lies on its biosynthetic pathway that is largely channeled towards the formation of polyglycosylated derivatives that are highly polar and water-soluble. These characteristics are attractive for extraction and potential use as food colorants, as well as for pharmacological uses [1].

In spite of constant extraction temperature, it is possible that this variable causes an increase of antioxidant compound solubility and thus, the liquid solvent diffusion coefficient in the solid matrix favors the desorption kinetics of matrix compounds [24]. However, high temperature has a strong influence on deployment of anthocyanins [29] and degradation of some phenolic glycosides and flavonols at higher temperatures [8]. In this experiment, the variable "temperature" was kept constant at $30^{\circ} \mathrm{C}$, so as not affect these bioactive compounds. 
It is known that the extraction time is an important parameter in the extraction procedure. According to the many authors, the extraction time can either be as short as a few minutes or very long, up to $24 \mathrm{~h}$. In this study, the range of extraction time was established based on practical and economical aspects (15-240 min). Heras et al. [30] mentioned that in anthocyanin extraction (main antioxidant compounds in berries) after an over prolonged time it could not be significant to pigment extraction. The Fick's diffusion law predicted that after certain time there will be a final equilibrium between the matrix solutes and the extraction solvent. The coefficient plot indicates that the extraction time range used in this experiment showed a non-significant impact on ORAC values (light brown bar in Figure 2).

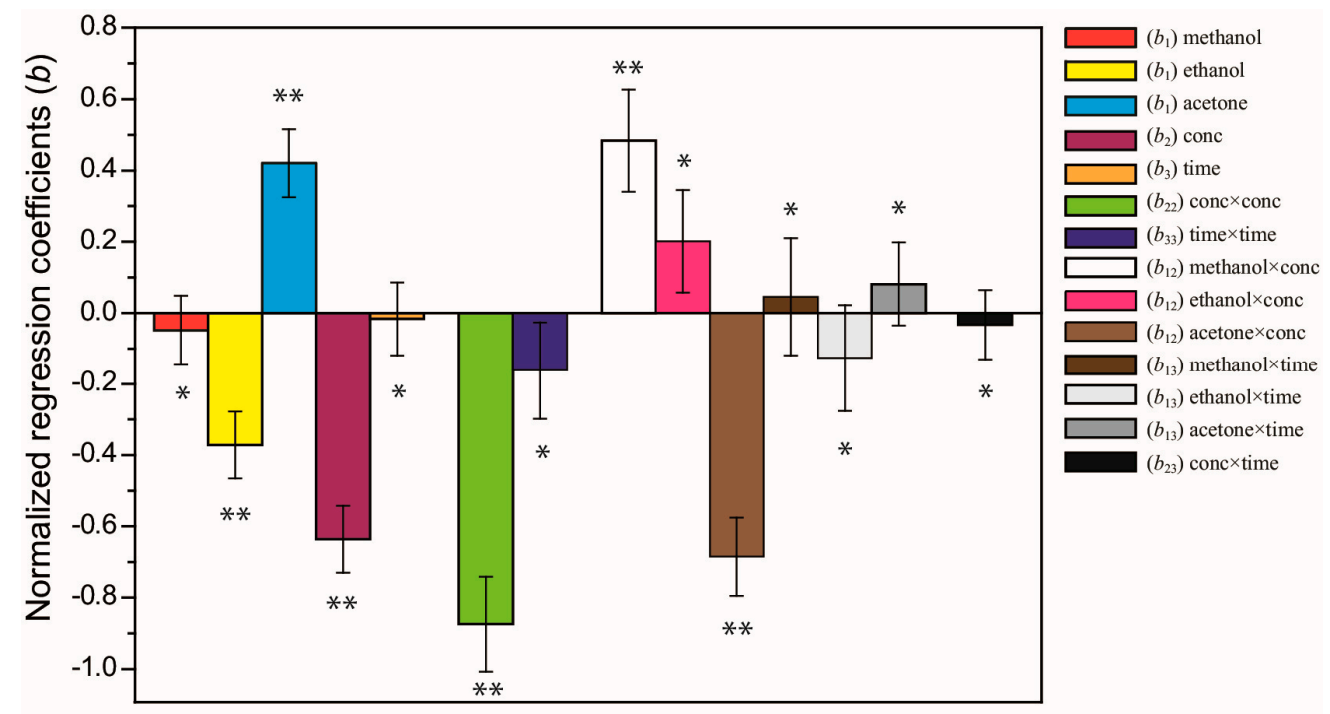

Figure 2. Coefficients plot of the model. The significance of the coefficients are $\left.{ }^{*}\right) p>0.05$ and $\left(^{* *}\right) p<0.05$.

By considering the regression coefficients obtained for independent variables, solvent type and solvent concentration were the most important factors that may significantly influence ORAC value. Similarly, Liyana-Pathirana \& Shahidi [31] reported that "solvent concentration" was the most important factor contributing to the extraction of phenolic compounds from wheat; and it was the "solvent" the one that had an influence on antioxidants from Borage leaves according Segovia et al. [8]. The large and negative interaction term $\left(b_{12}\right)$ of acetone $\times$ concentraction (brown bar in Figure 2) indicates that when acetone in used as solvent, the ORAC value increases when only a low concentration of solvent is used. However, a decreasing in ORAC values is obtained when acetone is used at high concentrations. The large and positive interaction term $\left(b_{12}\right)$ of methanol $\times$ concentraction (white bar in Figure 2) indicates that when methanol in used as solvent, the ORAC value increases when a high concentration of solvent is used and decreases when low concentrations are used. However, this interaction term is smaller than acetone $\times$ concentraction in magnitude. Thus, an optimal solvent and its concentration promote the extraction of specific substances of maqui berry and have a significant impact on the estimation of antioxidant capacity.

The ORAC value of maqui berry was optimized through RSM approach with SIMPLEX method. To visualize the overall effect of independent variables on ORAC value, 3D response surface maps of the quadratic polynomial were generated by varying two of the independent variables and keeping the solvent type constant as shown in Figure 3. These maps illustrate how ORAC behaves along with the variation of the percentage of solvent concentration scavenged $(20 \%-100 \%(v / v))$ and the variation of extraction time (15-240 $\mathrm{min}$ ) regarding to each solvent. In the case of acetone (Figure 3c), it was observed that using lower acetone concentrations, the higher the ORAC value, thus from range $20 \%-50 \%(v / v)$ acetone a higher ORAC was observed, independent of the extraction time used. 

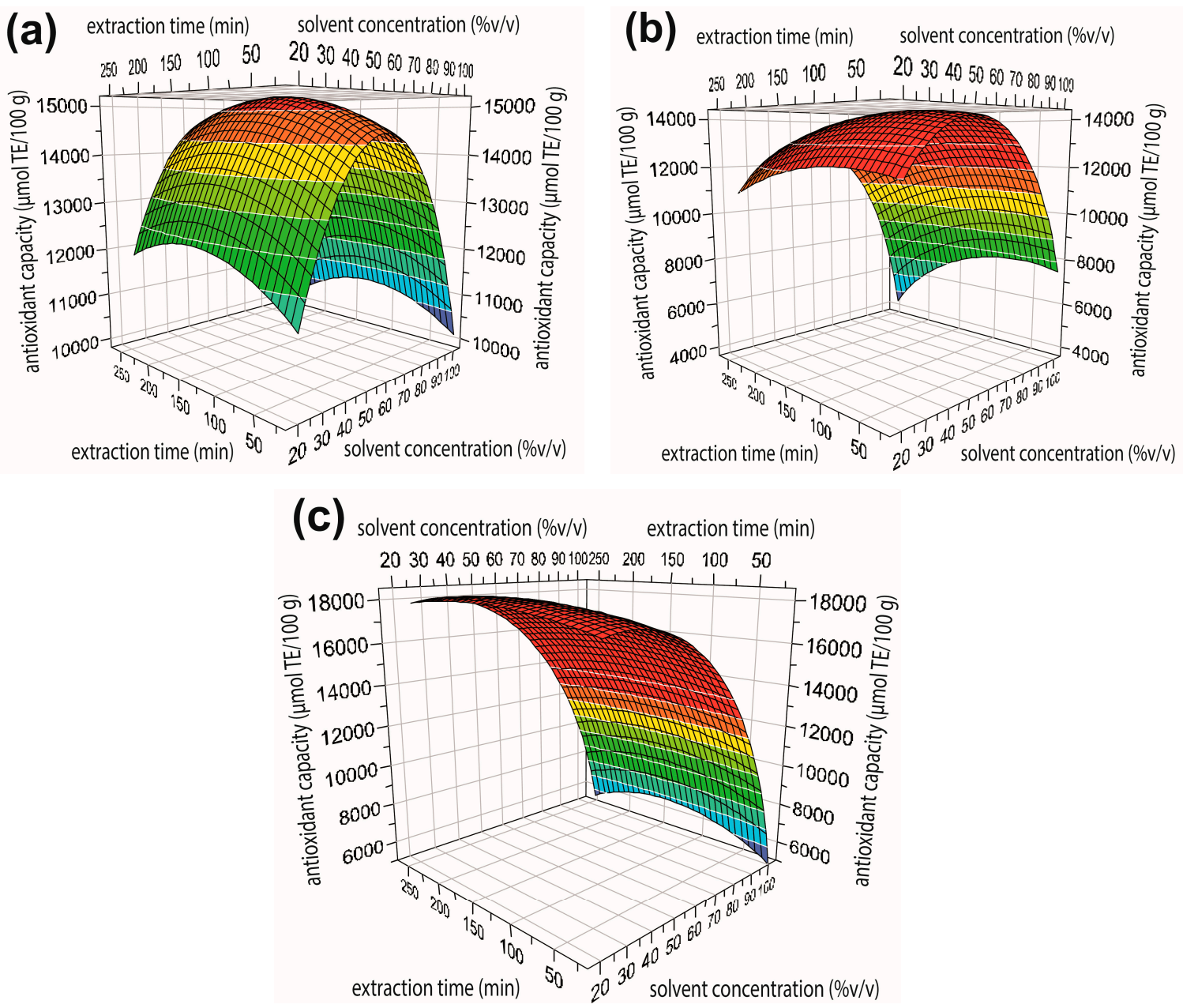

Figure 3. Response surface maps plotting $x_{2}$ solvent concentration vs. $x_{3}$ extraction time, with (a) $x_{1}$ methanol; (b) $x_{1}$ ethanol; and (c) $x_{1}$ acetone.

In the case of ethanol (Figure $3 b)$ it is shown that in a range of $40 \%-60 \%(v / v)$ the ORAC value is maximized. Figure 3a shows that the range of $40 \%-80 \%(v / v)$ methanol results in the highest ORAC values for this solvent. Along all extraction time range studied, no significant influence on the ORAC value for the three solvents is observed. Therefore, it could be backed up that extraction treatment at high temperatures and prolonged time causes a decrease on the antioxidant capacity according to Heras et al. [30], due to the long extraction process period that could be give rise to phenolic compounds oxidation because of the light, the exposition to oxygen and degradation. It seems that extraction time in berries of smaller diameter (4-6 $\mathrm{mm}$ approx.) does not significantly influence the antioxidant extraction, and therefore an adequate use of factor "time" would help to optimize the energy on the extracting processes.

Based on data collected from the experiment, the ORAC value was subjected to an optimization study with the aim of determining the optimum condition for the extraction of antioxidants from maqui berry. The optimal conditions that provide a maximum ORAC value are presented in Table 6 . Comparing the predicted response of ORAC value, an error lower than $1 \%$ was found respect the experimental value obtained from a sample performed with these optimum conditions. 
Table 6. Optimal variable settings for maximum antioxidant capacity response.

\begin{tabular}{lc}
\hline Variables & Value \\
\hline$x_{1}$ Type of solvent & Acetone \\
$x_{2}$ Solvent concentration $(\% v / v)$ & 29.13 \\
$x_{3}$ Extraction time $(\min )$ & 159.3 \\
$y$ Predicted response $(\mu \mathrm{mol} \mathrm{TE} / 100 \mathrm{~g})$ & $18,289.6$ \\
$y$ Experimental response $(\mu \mathrm{mol} \mathrm{TE} / 100 \mathrm{~g})$ & $18,137.6$ \\
\hline
\end{tabular}

\section{Conclusions}

A second-order polynomial model could be used to optimize extraction of phenolic compounds from maqui berry for maximizing the total antioxidant capacity (ORAC value). Aqueous acetone was found to be the most effective solvent to extract antioxidant compounds, being more efficient than methanol and ethanol. No-significant impact was found on the response for "extraction time" variable. The optimization model showed that the extraction conditions with 29\% (v/v) acetone and $159 \mathrm{~min}$ at $30{ }^{\circ} \mathrm{C}$ under agitation displayed the highest contents of ORAC value for fresh maqui. Statistical tools used, such as D-optimal design and RSM, allowed performing a suitable experiment to extract antioxidants from maqui berry. From the results above it can be concluded that the predictive model here described was a very well fitted antioxidant extracting process, according to the analyses of variance (ANOVA) and they can be applied to allow a fast, quantitative and maximum extraction of antioxidant compounds from maqui berry.

Acknowledgments: The authors appreciate financial support from the Research Office of La Serena University (DIULS, project PR15334) and CONICYT-PCHA/ National Doctorate /2016-21161653.

Author Contributions: Issis Quispe-Fuentes performed the experiments and wrote the paper. Víctor Campos-Requena designed the experiments and reviewed the paper. Antonio Vega-Gálvez contributed reagents, analysis tools and reviewed the paper.

Conflicts of Interest: The authors declare no have conflict of interest.

\section{References}

1. Escribano-Bailón, M.T.; Alcalde-Eon, C.; Muñoz, O.; Rivas-Gonzalo, J.C.; Santos-Buelga, C. Anthocyanins in Berries of Maqui [Aristotelia chilensis (Mol.) Stuntz]. Phytochem. Anal. 2006, 14, 8-14. [CrossRef]

2. Brauch, J.E.; Buchweitz, M.; Schweiggert, R.M.; Carle, R. Detailed analyses of fresh and dried maqui (Aristotelia chilensis (Mol.) Stuntz) berries and juice. Food Chem. 2016, 190, 308-316. [CrossRef] [PubMed]

3. Miranda-Rottmann, S.; Aspillaga, A.; Pérez, D.; Vasquez, L.; Martinez, A.; Leighton, F. Juice and Phenolic Fractions of the Berry Aristotelia chilensis Inhibit LDL Oxidation in Vitro and Protect Human Endothelial Cells against Oxidative Stress. J. Agric. Food Chem. 2002, 50, 7542-7547. [CrossRef] [PubMed]

4. Gironés-Vilaplana, A.; Baenas, N.; Villaño, D.; Speisky, H.; García-Viguera, C.; Moreno, D. Evaluation of Latin-American fruits rich in phytochemicals with biological effects. J. Funct. Foods 2014, 7, 599-608. [CrossRef]

5. Cespedes, L.C.; Alarcon, J.; Avila, J.G.; Nieto, A. Anti-inflammatory Activity of Aristotelia chilensis Mol. (Stuntz) (Elaeocarpaceae). Boletín Latinoam Y Del Caribe Plantas Med Y Aromáticas 2010, 9, 91-99.

6. González, B.; Vogel, H.; Razmilic, I.; Wolfram, E. Polyphenol, anthocyanin and antioxidant content in different parts of maqui fruits (Aristotelia chilensis) during ripening and conservation treatments after harvest. Ind. Crops Prod. 2015, 76, 158-165. [CrossRef]

7. Brigatto Fontes, L.C.; Ferraz da Silva Torres, A.E.; Yonekura, L. Optimization of the extraction of antioxidants from guarana (Paullinia cupana) and grape (Vitis labrusca var. Izabel ) pomace using response surface methodology. Afr. J. Food Sci. Technol. 2014, 5, 53-59.

8. Segovia, F.; Lupo, B.; Peiró, S.; Gordon, M.H.; Almajano, M.P. Extraction of Antioxidants from Borage (Borago officinalis L.) Leaves-Optimization by Response Surface Method and Application in Oil-in-Water Emulsions. Antioxidants 2014, 3, 339-357. [CrossRef] [PubMed] 
9. Khoddami, A.; Wilkes, M.A.; Roberts, T.H. Techniques for Analysis of Plant Phenolic Compounds. Molecules 2013, 18, 2328-2375. [CrossRef] [PubMed]

10. Fredes, C.; Yousef, G.G.; Robert, P.; Grace, M.H.; Lila, M.A.; Gómez, M.; Gebauer, M.; Montenegro, G. Anthocyanin profiling of wild maqui berries (Aristotelia chilensis [Mol.] Stuntz) from different geographical regions in Chile. J. Sci. Food Agric. 2014, 94, 2639-2648. [CrossRef] [PubMed]

11. Diem, Q.; Elisa, A.; Tran-nguyen, P.L.; Huong, L.; Edi, F.; Ismadji, S. Effect of extraction solvent on total phenol content, total flavonoid content, and antioxidant activity of Limnophila aromatica. J. Food Drug Anal. 2014, 22, 296-302.

12. Campos-Requena, V.H.; Rivas, B.L.; Perez, M.; Contreras, D.; Muñoz, E. Optimization of processing parameters for the synthesis of low-density polyethylene/organically modified montmorillonite nanocomposites using X-ray diffraction with experimental design. Polym. Int. 2013, 62, 548-553. [CrossRef]

13. Nwabueze, T.U. Review article: Basic steps in adapting response surface methodology as mathematical modelling for bioprocess optimisation in the food systems. Int. J. Food Sci. Technol. 2010, 45, 1768-1776. [CrossRef]

14. Ye, C.; Liu, X. Extraction of flavonoids from Tetrastigma Hemsleyanum Diels ET Gilg and their antioxidant activity. J. Food Process. Preserv. 2015, 39, 2197-2205. [CrossRef]

15. Ferri, M.; Gianotti, A.; Tassoni, A. Optimisation of assay conditions for the determination of antioxidant capacity and polyphenols in cereal food components. J. Food Compos. Anal. 2013, 30, 94-101. [CrossRef]

16. Saha, J.; Debnath, M.; Saha, A.; Ghosh, T.; Sarkar, P.K. Response surface optimisation of extraction of antioxida;nts from strawberry fruit, and lipid peroxidation inhibitory potential of the fruit extract in cooked chicken patties. J. Sci. Food Agric. 2011, 91, 1759-1765. [CrossRef] [PubMed]

17. Campos-Requena, V.H.; Rivas, B.L.; Pérez, M.A.; Garrido-Miranda, K.A.; Pereira, E.D. Polymer/clay nanocomposite films as active packaging material: Modeling of antimicrobial release. Eur. Polym. J. 2015, 71, 461-475. [CrossRef]

18. Shiby, V.K.; Radhakrishna, K.; Bawa, A.S. Development of whey-fruit-based energy drink mixes using D-optimal mixture design. Int. J. Food Sci. Technol. 2013, 48, 742-748. [CrossRef]

19. Puskas, V.; Miljic, U. The application of D-optimal design for modelling the red wine ageing process. Food Control 2012, 28, 362-367. [CrossRef]

20. Sproll, C.; Ruge, W.; Andlauer, C.; Godelmann, R.; Lachenmeier, D.W. HPLC analysis and safety assessment of coumarin in foods. Food Chem. 2008, 109, 462-469. [CrossRef] [PubMed]

21. Mannarswamy, A.; Munson-McGee, S.H.; Andersen, P.K. D-optimal designs for the Cross viscosity model applied to guar gum mixtures. J. Food Eng. 2010, 97, 403-409. [CrossRef]

22. Balsa-Canto, E.; Rodriguez-Fernandez, M.; Banga, J.R. Optimal design of dynamic experiments for improved estimation of kinetic parameters of thermal degradation. J. Food Eng. 2007, 82, 178-188. [CrossRef]

23. Peerapattana, J.; Ngamsupsiri, T.; Cheucharoenvasuchai, N.; Saikaew, C. Optimization of metronidazole sustained-release films using D-optimal design. Int. J. Pharm. 2015, 484, 1-7. [CrossRef] [PubMed]

24. Cacace, J.E.; Mazza, G. Optimization of Extraction of Anthocyanins from Black Currants with Aqueous Ethanol. J. Food Sci. 2003, 68, 240-248. [CrossRef]

25. Zhang, L.; Li, J.; Hogan, S.; Chung, H.; Welbaum, G.E.; Zhou, K. Inhibitory effect of raspberries on starch digestive enzyme and their antioxidant properties and phenolic composition. Food Chem. 2010, 119, 592-599. [CrossRef]

26. Eriksson, L.; Johansson, E.; Kettaneh-Wold, N.; Wikström, C.; Wold, S. Design of Experiments: Principles and Applications, 3rd ed.; Umetrics Academy: Umea, Sweden, 2000.

27. Rojo, L.E.; Ribnicky, D.; Logendra, S.; Poulev, A.; Rojas-Silva, P.; Kuhn, P.; Dorn, R.; Grace, M.H.; Raskin, I. In vitro and in vivo anti-diabetic effects of anthocyanins from Maqui Berry (Aristotelia chilensis). Food Chem. 2012, 131, 387-396. [CrossRef] [PubMed]

28. Arancibia-Avila, P.; Namiesnik, J.; Toledo, F.; Werner, E.; Martinez-Ayala, A.L.; Rocha-Guzmán, N.E.; Gallegos-Infante, J.A.; Gorinstein, S. The influence of different time durations of thermal processing on berries quality. Food Control 2012, 26, 587-593. [CrossRef]

29. Sadilova, E.; Stintzing, F.C.; Kammerer, D.R.; Carle, R. Matrix dependent impact of sugar and ascorbic acid addition on color and anthocyanin stability of black carrot, elderberry and strawberry single strength and from concentrate juices upon thermal treatment. Food Res. Int. 2009, 42, 1023-1033. [CrossRef] 
30. Heras, I.; Alvis, A. Optimización del Proceso de Extracción de Antocianinas y Evaluación de la Capacidad Antioxidante de Berenjena (Solana melonera L.) Optimization of the Anthocyanin Extraction Process and Evaluation of the Antioxidant Capacity of Eggplant (Solanum melong). Inf. Tecnol. 2013, 24, 93-102. [CrossRef]

31. Liyana-Pathirana, C.; Shahidi, F. Optimization of extraction of phenolic compounds from wheat using response surface methodology. Food Chem. 2005, 93, 47-56. [CrossRef] 